\title{
GW23-e1730 ASSESSMENT OF CORONARY ARTERY IN-STENT PATENCY BY FLASH-DSCT VERSUS CORONARY ARTERY ANGIOGRAPHY
}

\author{
doi:10.1136/heartjnl-2012-302920ad.16
}

Yang Jun-jie, Yang Xia, Du Luo-shan, Chen Yun-dai. Department of Cardiology, Chinese PLA General Hospital

Objectives Coronary computed tomographic modality sheds more light on radiation reduction while maintaining image quality. The aim of this study was to evaluate the performance of second generation dual source coronary CT scanner with prospective electrocardiographically triggered high-pitch spiral acquisition (FLASHDSCT) in the detection of instent restenosis (>50\% luminal narrowing) in symptomatic patients referred for conventional coronary angiography (CCA).

Methods 62 patients/107 stents with chest discomfort were prospectively evaluated after coronary stenting. Before CCA, FLASHCT was performed by using a second generation, dual-source CT scanner (Definition Flash; Siemens Healthcare, Forchheim, Germany) between September 2011 and March 2012.

Results Average heart rate (HR) was $58 \pm 7 \mathrm{bpm}$, and Average effective dose (ED) was $1.61 \pm 0.62 \mathrm{mSv}$. The interval between stenting and inclusion in the study was $31 \pm 40$ months. Of the 107 stents, $96(89.7 \%)$ could be assessed and $11(10.3 \%)$ were excluded due to unqualified image quality. $27 / 107(25.2 \%)$ stents had angiographically proven restenosis, and 46/107 (43\%) stents mild late loss. Sensitivity, specificity, positive predictive value (PPV) and negative predictive value (NPV) of FLASH-DSCT, calculated in all stents, were $95 \%, 93 \%, 76 \%$ and $98 \%$, respectively. In stents $>=3.5 \mathrm{~mm}$ $(\mathrm{n}=42)$, sensitivity, specificity, PPV, NPV were $100 \%$; in $3 \mathrm{~mm}$ stents $(n=39)$, sensitivity and NPV were $100 \%$, specificity $97 \%$, PPV $93 \%$; in stents $\leq 2.75 \mathrm{~mm}(\mathrm{n}=26)$, sensitivity was $88 \%$, specificity $66 \%$, PPV $59 \%$, NPV $90 \%$. Five stents $\leq 2.5 \mathrm{~mm}$ were indiscernible.

Conclusions It can be performed well in the detection of in-stent patency by FLASH-DSCT. In spite of frequent false positive findings in smaller stents $(\leq 2.75 \mathrm{~mm})$, FLASH-DSCT can reliably distinguishes in-stent restenosis irrespective of stent size. 